\title{
Risk Factors for Short-Term Virologic Outcomes Among HIV-Infected Patients Undergoing Regimen Switch of Combination Antiretroviral Therapy
}

\author{
Chun Chao, Beth Tang,, Leo Hurley, ${ }^{1}$ Michael J Silverberg, ${ }^{2}$ William Towner, ${ }^{3}$ \\ Melissa Preciado, and Michael Horberg ${ }^{4}$
}

\begin{abstract}
We investigated risk factors for unfavorable virologic responses among HIV-infected patients who recently switched antiretroviral regimens. We identified HIV-infected patients who switched antiretroviral regimens (defined as adding $\geq 2$ new medications) between 2001 and 2008 at Kaiser Permanente California. Virological response, measured after 6 months on the new regimen, was classified as (1) maximal viral suppression (HIV RNA $<75 / \mathrm{ml}$ ), (2) low-level viremia (LLV; 75-5000/ml), or (3) advanced virologic failure ( $>5000 / \mathrm{ml})$. Potential risk factors examined included (1) HIV disease factors, e.g., prior AIDS, CD4 cell count; (2) history of antiretroviral use, e.g., therapy classes of the newly switched regimen, medication adherence, and virologic failure at previous regimens; and (3) novel patient-level factors including comorbidities and healthcare utilization. Adjusted odds ratios (aOR) for LLV and advanced virologic failure were obtained from multivariable nominal logistic regression models. A total of 3447 patients were included; 2608 (76\%) achieved maximal viral suppression, $420(12 \%)$ had LLV, and $419(12 \%)$ developed advanced virologic failure. Factors positively associated with LLV and advanced virologic failure included number of regimens prior to switch $\left[\mathrm{aOR}_{\text {per regimen }}=1.38\right.$ (1.17-1.62) and 1.77 (1.50-2.08), respectively], nucleotide reverse transcriptase inhibitor-only regimens (vs. protease inhibitor-based) $[\mathrm{aOR}=2.78(1.28-6.04)$ and $5.10(2.38-10.90)$, respectively], and virologic failure at previous regimens $[\mathrm{aOR}=3.15$ (2.17-4.57) and 4.71 (2.84-7.81), respectively]. Older age, higher CD4 cell count, and medication adherence were protective for unfavorable virologic outcomes. Antiretroviral regimen-level factors and immunodeficiency were significantly associated with virologic failure after a recent therapy switch and should be considered when making treatment change decisions.
\end{abstract}

\section{Introduction}

T HE THERAPEUTIC MANAgEMENT OF $\mathrm{HIV}^{+}$patients involves the balance of maintaining maximally controlled HIV RNA levels and high CD4 T cell counts, while minimizing adverse effects of combination antiretroviral therapy (cART). Current treatment guidelines for HIV patients advocate targeting maximal viral control and early regimen switch in the event of treatment failure. ${ }^{1}$ However, not all patients are able to achieve and maintain HIV RNA level below the assay's lower limits of quantification, whether it is due to poor absorption, poor adherence, or viral resistance. The choice of antiretroviral medication ultimately becomes limited and often requires complex regimens with potentially greater pill burden or adverse effects. Failure to achieve maximal viral suppression thus poses challenges to the clinical management of $\mathrm{HIV}^{+}$patients, particularly among those who have been previously treated with multiple antiretrovirals.

Information on predictors of virologic outcome among treatment-experienced patients therefore would be helpful for clinicians considering regimen changes. For example, particularly high-risk subpopulations could be targeted for close monitoring and adherence counseling. Previous studies reported that several factors may influence the likelihood of achieving maximal viral control after switching regimens, including demographics, ${ }^{2,3}$ previous virologic response, ${ }^{2,4-6}$

\footnotetext{
${ }^{1}$ Department of Research and Evaluation, Kaiser Permanente Southern California, Pasadena, California

${ }^{2}$ Division of Research, Kaiser Permanente Northern California, Oakland, California.

${ }^{3}$ Los Angeles Medical Center, Kaiser Permanente Southern California, Los Angeles, California.

${ }^{4}$ Mid-Atlantic Permanente Research Institute, Kaiser Permanente Mid-Atlantic, Rockville, Maryland.
} 
previous exposure to antivirals, ${ }^{7-9}$ and medication adherence. ${ }^{10,11}$ However, many studies did not separately examine failing with low-level viremia (LLV) from advanced virologic failure. Previous studies reported that clinical outcomes vary for those failing with LLV vs. those with advanced virologic failure. ${ }^{12,13}$ Specifically, failing with LLV may not translate into accelerated disease progression. ${ }^{12-14}$ Therefore, understanding risk factors specifically for advanced virologic failure vs. failing with LLV may provide clinicians with useful information for managing treatment-experienced HIV-infected patients. In this study, we investigated risk factors for advanced virologic and for LLV among $\mathrm{HIV}^{+}$ patients who underwent cART regimen switch in Kaiser Permanente California health plans. We examined the role of HIV disease factors, such as CD4 cell count and prior AIDS diagnosis, antiretroviral regimen-level factors, as well as novel patient-level factors, such as comorbidity and healthcare utilization, for these unfavorable virologic outcomes.

\section{Materials and Methods}

\section{Study population}

Kaiser Permanente (KP) California Health Plans are large integrated healthcare delivery systems in California, serving over 6.6 million racial/ethnically and socioeconomically diverse members who are broadly representative of the California insured population. The health plans have maintained active HIV patient registries. $\mathrm{HIV}^{+}$patients included in these HIV registries were initially identified using electronic health records, and confirmed as true cases by chart review and/or consulting with clinical staff at the patient's medical centers.

$\mathrm{HIV}^{+}$persons age 18 and older who underwent a cART regimen switch (defined as changing of at least two antiretroviral medications) between January 2001 and June 2008 constituted our subjects of interest. For each person, his or her clinical experience on the first regimen switch that met the following criteria was included in the analysis: (1) there was at least 6 months of health plan membership prior to the regimen switch; (2) the person remained on the newly switched regimen for at least 6 months, i.e., the stabilization period, without switching to another regimen; and (3) there was an HIV RNA measurement (see Outcome Assessment below) to allow assessment of virologic response on the new regimen. The 6-month prior membership criterion was required to assess the person's clinical history. The requirement of the 6-month stabilization period on the new regimen was to assess treatment response on the new regimen beyond initial virologic adjustment after regimen switch. This study was approved by the responsible Institutional Review Boards and the requirement for informed consent was waived.

\section{Data collection}

Measurements of covariates. The following covariates measured at time of regimen switch were examined as potential risk factors for failing on the new regimen: (1) demographic characteristics, including age, gender, race/ethnicity, and public insurance (Medicare and Medicaid) status; (2) HIV disease factors, including HIV transmission risk group, years of known HIV infection in KP, prior AIDS-defining diagnosis, and CD4 cell count at time of regimen switch; (3) cART use history, including known years of cART use at KP, HIV genotyping done immediately prior to regimen switch (yes/ no), ART class of the new regimen [protease inhibitor (PI)based, nonnucleotide reverse transcriptase inhibitor (NNRTI)based, nucleotide reverse transcriptase inhibitor (NRTI)-only, new class (i.e., integrase inhibitor and entry inhibitor), and mixed class], number of cART regimens ever taken, and virologic failure at the previous regimen (not all subjects had evidence of virologic failure at the time of regimen switch); (4) other patient-level factors, including comorbidities such as history of cardiovascular disease, hypertension, diabetes mellitus, obesity, hepatitis B and C infection, and non-AIDS defining cancer; and healthcare utilization, such as number of office visit, emergency room visit, and hospitalization in the 6 months prior to regimen switch; and (5) calendar year of the regimen switch. In addition, cART adherence to the newly switched, current regimen (referred as the "new regimen" in the following text) during the 6-month stabilization period was also assessed.

Data for all covariates were collected from KP's disease registries and electronic medical records. Age, gender, race/ ethnicity, years of known HIV infection, HIV transmission risk group, and AIDS-defining illnesses were obtained from KP's HIV registries. Use of cART, defined as three or more antiretrovirals, was captured by KP's pharmacy databases, which includes prescriptions dispensed at all KP medical offices. Over $95 \%$ of KP members have a drug benefit and fill their prescriptions at KP pharmacies (including ADAPeligible prescriptions). Adherence to the cART regimen was assessed as a variable ranged between $0 \%$ and $100 \%$ and was calculated by taking the total number of days covered by a patient's filled prescriptions divided by the total number of days in the specified period. CD4 cell count measurements were obtained from the KP laboratory database. Comorbid conditions were assessed using inpatient/outpatient ICD-9 diagnosis codes, coupled with laboratory tests for the hepatitis B/C serology. History of cancers was assessed using KP California's Surveillance, Epidemiology and End Results (SEER)-affiliated cancer registries. Healthcare utilization was assessed using the health plan's outpatient and inpatient utilization files.

Outcome assessment. The outcome of interest was achieving maximal viral suppression (defined as HIV RNA $<75$ copies/ml), LLV (defined as $75 \leq$ HIV RNA $\leq 5000$ copies $/ \mathrm{ml}$ ), or advanced virologic failure (defined as HIV RNA $>5000$ copies $/ \mathrm{ml})$ at 6 months ( \pm 8 weeks) after initiating the new regimen. Two levels of treatment failure were considered, i.e., LLV and advanced virologic failure, because studies reported that clinical outcomes vary for those failing with LLV vs. those with advanced virologic failure. ${ }^{12,13}$ HIV RNA measurements were obtained from the Kaiser Permanente's laboratory database.

\section{Statistical analysis}

We calculated the distributions of covariates by HIV virologic response on the new regimen. Associations between covariates and HIV virologic response were tested using the chi-square test for categorical variables and Kruskal-Wallis test for continuous variables. Next, crude and adjusted associations between these factors and HIV virologic response were evaluated in logistic regression models. Age, gender, 
race/ethnicity, KP region (northern or southern California), HIV transmission risk group, and cART class of the new regimen were specified a priori to be included in the multivariable analysis, regardless of statistical significance. In addition, covariates that demonstrated a $p$-value $<0.10$ in the univariate analysis were included in the final model. We also conducted stratified analysis by CD4 cell count at regimen switch of $<200 / \mu \mathrm{l}$ and $\geq 200 / \mu \mathrm{l}$. In a sensitivity analysis, we restricted the analysis to $52 \%$ of the study subjects for whom data on first date of HIV diagnosis and date of first cART use prior to their KP membership were available. Adjusting for the total duration of know HIV infection and cART use (including pre-KP data), the results are similar to that in the unrestricted analysis. Therefore, results from the unrestricted analysis that includes all subjects were presented. All analyses were conducted using SAS statistical software version 9.2 (Statistical Analyses System Inc., Cary, NC).

\section{Results}

We identified a total of $4847 \mathrm{HIV}^{+}$patients at Kaiser Permanente California who were of age 18 years or older and had a cART regimen switch between 2001 and 2008. Among them, 4411 met the 6-month prior health plan membership inclusion criteria. Of these, 18 were excluded because they did not remain on the new regimen for at least 6 months (the stabilization period), and 946 were excluded due to the lack of an HIV RNA measurement within the specified time period for

Table 1. Subject Characteristics at Regimen Switch by HiV Virologic Response at the End of the Stabilization Period on the Current Regimen

\begin{tabular}{|c|c|c|c|c|c|}
\hline & $\begin{array}{c}\text { All } \\
(\mathrm{N}=3447)\end{array}$ & $\begin{array}{c}\text { Advanced treatment } \\
\text { failure of HIV } \\
\text { RNA }>5000 \\
\text { copies } / m l(\mathrm{~N}=419)\end{array}$ & $\begin{array}{c}\text { Low-level viremia of } \\
75<H I V \text { RNA } \leq 5000 \\
\text { copies/ml }(\mathrm{N}=420)\end{array}$ & $\begin{array}{c}\text { Maximal viral } \\
\text { suppression of } \\
\text { HIV RNA }<75 \\
\text { copies/ml }(\mathrm{N}=2608)\end{array}$ & \multirow[b]{2}{*}{$\mathrm{p}$-value } \\
\hline & \multicolumn{4}{|c|}{ Number $(\%)^{\mathrm{a}} /$ mean $(S D)$} & \\
\hline \multicolumn{6}{|l|}{ Demographics } \\
\hline Age [year, mean (SD)] & $47.5(9.4)$ & $45.0(8.8)$ & $46.0(9.0)$ & $48.1(9.5)$ & $<0.01$ \\
\hline Male gender & $3087(89.6 \%)$ & $374(89.3 \%)$ & $373(88.8 \%)$ & $2340(89.7 \%)$ & 0.80 \\
\hline \multicolumn{6}{|l|}{ Race/ethnicity } \\
\hline White & $1895(55.0 \%)$ & $187(44.6 \%)$ & $220(52.4 \%)$ & $1488(57.1 \%)$ & $<0.01$ \\
\hline Black & $612(17.8 \%)$ & $102(24.3 \%)$ & $78(18.6 \%)$ & $432(16.6 \%)$ & \\
\hline Hispanic & $706(20.5 \%)$ & $97(23.2 \%)$ & $96(22.9 \%)$ & $513(19.7 \%)$ & \\
\hline Asian/Pacific islander & $144(4.2 \%)$ & $20(4.8 \%)$ & $18(4.3 \%)$ & $106(4.1 \%)$ & \\
\hline Medicare enrollees & $664(19.3 \%)$ & $104(24.8 \%)$ & $83(19.8 \%)$ & $477(18.3 \%)$ & 0.01 \\
\hline Medicaid enrollees & $324(9.4 \%)$ & $62(14.8 \%)$ & $47(11.2 \%)$ & $215(8.2 \%)$ & $<0.01$ \\
\hline \multicolumn{6}{|l|}{ HIV disease factors } \\
\hline \multicolumn{6}{|l|}{ HIV transmission risk group } \\
\hline Men having sex with men & $2224(64.5 \%)$ & $247(59.0 \%)$ & $271(64.5 \%)$ & $706(65.4 \%)$ & 0.16 \\
\hline Intravenous drug users & $109(3.2 \%)$ & $17(4.1 \%)$ & $16(03.8 \%)$ & $76(2.9 \%)$ & \\
\hline Heterosexual & $483(14.0 \%)$ & $62(14.8 \%)$ & $60(14.3 \%)$ & $361(13.8 \%)$ & \\
\hline Other & $68(2.0 \%)$ & $7(1.7 \%)$ & $5(1.2 \%)$ & $56(2.2 \%)$ & \\
\hline Prior AIDS diagnosis ${ }^{\mathrm{b}}$ & $2148(62.3 \%)$ & $330(78.8 \%)$ & $280(66.7 \%)$ & $1538(59.0 \%)$ & $<0.01$ \\
\hline CD4 cell count $(/ \mu \mathrm{l})$ & $377.3(267.8)$ & $219.4(203.6)$ & $315.8(237.4)$ & $412.5(270.6)$ & $<0.01$ \\
\hline $\begin{array}{l}\text { Years of known HIV } \\
\text { infection in KP }\end{array}$ & $9.3(5.3)$ & $8.7(5.1)$ & $8.9(4.9)$ & $9.5(5.3)$ & 0.01 \\
\hline \multicolumn{6}{|l|}{ Comorbid conditions } \\
\hline Hepatitis B infection & $182(5.3 \%)$ & $29(6.9 \%)$ & $23(5.5 \%)$ & $130(5.0 \%)$ & 0.25 \\
\hline Hepatitis $C$ infection & $363(10.5 \%)$ & $37(8.8 \%)$ & $50(11.9 \%)$ & $276(10.6 \%)$ & 0.34 \\
\hline Diabetes mellitus & $390(11.3 \%)$ & $51(12.2 \%)$ & $42(10.0 \%)$ & $297(11.4 \%)$ & 0.59 \\
\hline Hypertension & $913(26.5 \%)$ & $89(21.2 \%)$ & $96(22.9 \%)$ & $728(27.9 \%)$ & $<0.01$ \\
\hline Non-AIDS-defining cancer & $175(5.1 \%)$ & $17(4.1 \%)$ & $22(5.2 \%)$ & $136(5.2 \%)$ & 0.60 \\
\hline Cardiovascular disease & $162(4.7 \%)$ & $16(3.8 \%)$ & $16(3.8 \%)$ & $130(5.0 \%)$ & 0.38 \\
\hline Obesity & $933(27.1 \%)$ & $69(16.5 \%)$ & $87(20.7 \%)$ & $777(29.8 \%)$ & $<0.01$ \\
\hline \multicolumn{6}{|c|}{ Health service utilization in the 6 months prior to regimen switch } \\
\hline Office visit, \# & $12.9(11.8)$ & $14.2(12.7)$ & $13.1(12.6)$ & $12.6(11.5)$ & 0.01 \\
\hline Emergency room visit, \# & $0.5(1.2)$ & $0.8(1.8)$ & $0.6(1.2)$ & $0.5(1.1)$ & $<0.01$ \\
\hline Hospitalization, \# & $0.2(0.7)$ & $0.3(1.0)$ & $0.2(0.8)$ & $0.2(0.6)$ & 0.01 \\
\hline \multicolumn{6}{|l|}{ Calendar year at regimen switch } \\
\hline $2001-2002$ & $810(23.5 \%)$ & $179(42.7 \%)$ & $165(39.3 \%)$ & $466(17.9 \%)$ & $<0.01$ \\
\hline 2003-2004 & $984(28.5 \%)$ & $132(31.5 \%)$ & $130(31.0 \%)$ & $722(27.7 \%)$ & \\
\hline 2005-2006 & $981(28.5 \%)$ & 79 (18.9\%) & 77 (18.3\%) & $825(31.6 \%)$ & \\
\hline 2007-2008 & $672(19.5 \%)$ & $29(6.9 \%)$ & $48(11.4 \%)$ & $595(22.8 \%)$ & \\
\hline
\end{tabular}

apercent may not add up to $100 \%$ due to missing data.

${ }^{\mathrm{b}}$ AIDS diagnosis was based on the Centers for Disease Control and Prevention 1993 clinical AIDS criteria, except that the criterion of CD4 cell count less than $200 / \mu$ l was not used. 
determining HIV virologic response on the new regimen. A total of 3447 subjects were included in the study. At the end of the stabilization period, $2608(76 \%)$ subjects achieved maximal viral suppression, 420 (12\%) failed with LLV, and 419 (12\%) experienced advanced virologic failure with HIV RNA > 5000 copies $/ \mathrm{ml}$.

Subject characteristics at the time of the regimen switch are shown in Table 1 . The study population was mostly male $(89.6 \%)$, of white race $(55.0 \%)$, and were men who have sex with men (MSM, 64.5\%), consistent with the HIV epidemic in California. The study population had a mean exposure to 2.4 regimens (Table 2). Those who developed treatment failure on the new regimen were on average younger, more likely to be racial/ethnic minority, and more likely to be Medicare/ Medicaid enrollees. In the crude analyses, all covariates examined, except gender, HIV transmission risk group, years of known cART use, and several comorbid conditions, were significantly associated with unfavorable HIV virologic responses.

Table 3 presents the covariates included in the multivariable model and their adjusted odds ratios (OR) and 95\% confidence intervals. Younger age was associated with both advanced virologic failure and failing with LLV on the new regimen. Subjects who were heterosexual [OR $=1.56(0.99-$ 2.46), compared with MSM] as well as those with lower CD4 cell count $\left[\mathrm{OR}=0.82(0.76-0.89)\right.$ per $100 / \mathrm{mm}^{3}$ increase] had elevated odds of developing advanced virologic failure. No association was observed with history of comorbidity or healthcare utilization. Advancement in calendar year was associated with decreased likelihood of treatment failure (Table 3).

With regard to the cART regimen-level factors, regimen count and therapy class of the new regimen were both significantly associated with the odds of treatment failure (Table 3). Comparing with PI-based regimens, NRTI-only regimens were associated with both failing with advanced virologic failure and with LLV. New class (integrase inhibitors and entry inhibitors)-based and mixed class-based regimens, on the other hand, appear to be protective for failing with LLV. As expected, virologic failure at previous regimen was a strong risk factor for failing the new regimen $[\mathrm{OR}=4.71$ (2.84-7.81) for advanced failure and 3.15 (2.17-4.57) for LLV], and greater medication adherence to the new regimen was protective for failing with both advanced virologic failure and LLV [OR $=0.96$ (0.95-0.97) for advanced failure and 0.97 (0.96-0.98) for LLV per $1 \%$ increase in adherence] (Table 3 ).

When we repeated the analyses stratified by CD4 cell count at regimen switch, the same risk factors were identified for treatment failure among the group with CD4 cell count $<200 / \mu$ l and the group with CD4 cell count $\geq 200 / \mu$ l. However, it should be mentioned that among those with CD4 cell count of $200 / \mu \mathrm{l}$ and higher, new class-based cART regimen

Table 2. Combination Antiretroviral Therapy (cART) Use Among Study Subjects

\begin{tabular}{|c|c|c|c|c|c|}
\hline & $\begin{array}{c}A l l \\
(\mathrm{~N}=3447)\end{array}$ & $\begin{array}{l}\text { Advanced treatment } \\
\text { failure of HIV } \\
\text { RNA }>5000 \\
\text { copies/ml }(\mathrm{N}=419)\end{array}$ & $\begin{array}{c}\text { Low-level viremia } \\
\text { of } 75<H I V \\
\text { RNA } \leq 5000 \\
\text { copies/ml }(\mathrm{N}=420)\end{array}$ & $\begin{array}{c}\text { Maximal viral } \\
\text { suppression of } \\
\text { HIV RNA }<75 \\
\text { copies/ml }(\mathrm{N}=2608)\end{array}$ & \multirow[b]{2}{*}{$\mathrm{p}$-value } \\
\hline & \multicolumn{4}{|c|}{ Number $(\%) /$ mean $(S D)$} & \\
\hline Years of known cART use (year) & $7.4(4.6)$ & $7.1(6.1)$ & $7.2(4.2)$ & $7.4(4.4)$ & 0.13 \\
\hline $\begin{array}{l}\text { Number of regimen count } \\
{[\text { mean (SD) }]^{a}}\end{array}$ & $2.4(0.7)$ & $2.7(0.9)$ & $2.5(0.8)$ & $2.3(0.6)$ & $<0.01$ \\
\hline \multicolumn{6}{|l|}{ Therapy class of the current regimen } \\
\hline PI based & $1291(37.5 \%)$ & $176(42.0 \%)$ & $181(43.1 \%)$ & $934(35.8 \%)$ & \\
\hline NNRTI based & $827(24.0 \%)$ & $67(16.0 \%)$ & $86(20.5 \%)$ & $674(25.8 \%)$ & \\
\hline NRTI based & $54(1.6 \%)$ & $21(5.0 \%)$ & $14(3.3 \%)$ & $19(0.7 \%)$ & $<0.01$ \\
\hline New class ${ }^{b}$ & $228(6.6 \%)$ & $28(6.7 \%)$ & $16(3.8 \%)$ & $184(7.1 \%)$ & \\
\hline Mixed & $1047(30.4 \%)$ & $127(30.3 \%)$ & $23(29.3 \%)$ & $797(30.6 \%)$ & \\
\hline \multicolumn{6}{|c|}{ Therapy class of the regimen prior to regimen switch $^{c}$} \\
\hline PI based & $1245(36.1 \%)$ & $154(36.8 \%)$ & $168(40.0 \%)$ & $923(35.4 \%)$ & $<0.01$ \\
\hline NNRTI based & $949(27.5 \%)$ & $71(17.0 \%)$ & $96(22.9 \%)$ & $782(30.0 \%)$ & \\
\hline NRTI only & $128(3.7 \%)$ & $20(4.8 \%)$ & $17(4.1 \%)$ & $91(3.5 \%)$ & \\
\hline New class ${ }^{b}$ & $113(3.3 \%)$ & $4(1.0 \%)$ & $5(1.2 \%)$ & $104(4.0 \%)$ & \\
\hline Mixed & $262(7.6 \%)$ & $49(11.7 \%)$ & $31(7.4 \%)$ & $182(7.0 \%)$ & \\
\hline Non-cART & $750(21.8 \%)$ & $121(28.9 \%)$ & $103(24.5 \%)$ & $526(20.2 \%)$ & \\
\hline Virologic failure at previous regimen & $2281(66.2 \%)$ & $394(94.0 \%)$ & $364(86.7 \%)$ & 1523 (58.4\%) & $<0.01$ \\
\hline $\begin{array}{l}\text { HIV genotyping prior to } \\
\text { regimen switch }\end{array}$ & $1304(37.8 \%)$ & $205(48.9 \%)$ & $206(49.1 \%)$ & $893(34.2 \%)$ & $<0.01$ \\
\hline \multicolumn{6}{|c|}{ cART regimen adherence during the 6-month stabilization period } \\
\hline $\begin{array}{l}\text { Average \% days adherent to the } \\
\text { current regimen [mean (SD)] }\end{array}$ & 90.7 (12.9) & 82.8 (17.5) & $85.9(16.8)$ & 92.6 (10.6) & $<0.01$ \\
\hline
\end{tabular}

${ }^{\text {a }}$ Included the new regimen of the regimen switch.

${ }^{b} \mathrm{New}$ class includes integrase inhibitors and entry inhibitors.

${ }^{\mathrm{c}}$ Assessed based on antivirals used within 6 months prior to regimen switch.

${ }^{\mathrm{d}}$ Although subjects were not on cART within the 6 months prior to regimen switch, these subjects were previously on cART.

PI, protease inhibitor; NNRTI, nonnucleoside reverse transcriptase inhibitor; NRTI, nucleoside reverse transcriptase inhibitor. 
Table 3. Multivariable Nominal Logistic Regression for Viral Control Status at the End of the Stabilization Period on Index Regimen

\begin{tabular}{|c|c|c|c|c|}
\hline & \multicolumn{4}{|c|}{ Viral control status at the end of the stabilization period } \\
\hline & \multicolumn{2}{|c|}{$\begin{array}{l}\text { Advanced virologic failure of } \\
\text { HIV RNA }>5000 \text { copies } / \mathrm{ml}\end{array}$} & \multicolumn{2}{|c|}{$\begin{array}{c}\text { Low level viremia of } 75<H I V \\
\text { RNA }<5000 \text { copies } / \mathrm{ml}\end{array}$} \\
\hline & $\begin{array}{l}\text { Odds ratio }{ }^{\mathrm{a}}(95 \% \\
\text { confidence interval) }\end{array}$ & $\mathrm{p}$-value & $\begin{array}{l}\text { Odds ratio }{ }^{\mathrm{a}}(95 \% \\
\text { confidence interval) }\end{array}$ & p-value \\
\hline \multicolumn{5}{|l|}{ Demographics } \\
\hline Age (per 10 year increase) & $0.81(0.69-0.95)$ & $<0.01$ & $0.87(0.76-1.00)$ & 0.05 \\
\hline Male gender & $1.35(0.80-2.26)$ & 0.26 & $0.97(0.60-1.55)$ & 0.89 \\
\hline \multicolumn{5}{|l|}{ Race/ethnicity } \\
\hline Black vs. white & $1.28(0.92-1.78)$ & 0.14 & $0.94(0.68-1.29)$ & 0.69 \\
\hline Hispanic vs. white & $1.17(0.85-1.62)$ & 0.34 & $1.04(0.78-1.39)$ & 0.80 \\
\hline Asian/Pacific islander vs. white & $1.37(0.73-2.54)$ & 0.33 & $1.08(0.61-1.91)$ & 0.80 \\
\hline Medicare enrollees & $1.22(0.89-1.68)$ & 0.28 & $1.01(0.74-1.38)$ & 0.94 \\
\hline Medicaid enrollees & $1.24(0.84-1.84)$ & 0.28 & $1.12(0.76-1.64)$ & 0.58 \\
\hline \multicolumn{5}{|l|}{ HIV disease factors } \\
\hline \multicolumn{5}{|l|}{ HIV transmission risk group } \\
\hline Intravenous drug users vs. MSM & $1.33(0.69-2.54)$ & 0.40 & $1.18(0.64-2.18)$ & 0.60 \\
\hline Heterosexual vs. MSM & $1.56(0.99-2.46)$ & 0.05 & $1.15(0.75-1.77)$ & 0.53 \\
\hline Other vs. MSM & $0.81(0.32-2.06)$ & 0.66 & $0.46(0.17-1.24)$ & 0.13 \\
\hline Prior AIDS diagnosis & $1.18(0.81-1.70)$ & 0.39 & $1.01(0.76-1.35)$ & 0.95 \\
\hline CD 4 cell count (per $100 / \mu 1$ increase) & $0.82(0.76-0.89)$ & $<0.01$ & $0.99(0.93-1.05)$ & 0.62 \\
\hline $\begin{array}{l}\text { Known duration of HIV infection } \\
\text { in KP (per year increase) }\end{array}$ & $1.01(0.98-1.04)$ & 0.40 & $1.01(0.98-1.03)$ & 0.64 \\
\hline \multicolumn{5}{|l|}{ Comorbid conditions } \\
\hline Hypertension & $1.05(0.77-1.44)$ & 0.74 & $1.06(0.80-1.40)$ & 0.70 \\
\hline \multicolumn{5}{|c|}{ Health service utilization in 6 months prior to regimen switch } \\
\hline Office visit, \# & $1.01(1.00-1.02)$ & 0.34 & $1.00(0.99-1.01)$ & 0.43 \\
\hline Emergency room visit, \# & $1.08(0.97-1.19)$ & 0.15 & $1.02(0.91-1.14)$ & 0.75 \\
\hline Hospitalization, \# & $0.99(0.82-1.18)$ & 0.88 & $0.96(0.78-1.17)$ & 0.68 \\
\hline \multicolumn{5}{|l|}{ Calendar year at regimen switch } \\
\hline 2003-2004 vs. 2001-2002 & $0.65(0.48-0.88)$ & $<0.01$ & $0.61(0.46-0.80)$ & $<0.01$ \\
\hline 2005-2006 vs. $2001-2002$ & $0.47(0.33-0.67)$ & $<0.01$ & $0.38(0.27-0.53)$ & $<0.01$ \\
\hline $2007-2008$ vs. $2001-2002$ & $0.27(0.16-0.46)$ & $<0.01$ & $0.37(0.24-0.56)$ & $<0.01$ \\
\hline \multicolumn{5}{|l|}{ cART regimen level factors } \\
\hline Number of regimen count (per regimen increase) & $1.77(1.50-2.08)$ & $<0.01$ & $1.38(1.17-1.62)$ & $<0.01$ \\
\hline \multicolumn{5}{|l|}{ Therapy class of the current regimen } \\
\hline NNRTI based vs. PI based & $1.32(0.92-1.90)$ & 0.13 & $1.13(0.83-1.53)$ & 0.45 \\
\hline NRTI only vs. PI based & $5.10(2.38-10.90)$ & $<0.01$ & $2.78(1.28-6.04)$ & $<0.01$ \\
\hline New class ${ }^{b}$ vs. PI based & $0.63(0.36-1.10)$ & 0.10 & $0.42(0.23-0.78)$ & $<0.01$ \\
\hline Mixed vs. PI based & $0.88(0.65-1.18)$ & 0.38 & $0.71(0.54-0.93)$ & 0.01 \\
\hline Virologic failure at previous regimen & $4.71(2.84-7.81)$ & $<0.01$ & $3.15(2.17-4.57)$ & $<0.01$ \\
\hline HIV genotyping prior to regimen switch & $0.89(0.69-1.15)$ & 0.37 & $1.12(0.88-1.42)$ & 0.36 \\
\hline \multicolumn{5}{|l|}{ cART regimen adherence during the } \\
\hline $\begin{array}{l}\text { Average \% days adherent to the new } \\
\text { regimen (per } 1 \% \text { increase) }\end{array}$ & $0.96(0.95-0.97)$ & $<0.01$ & $0.97(0.96-0.98)$ & $<0.01$ \\
\hline
\end{tabular}

${ }^{a}$ Reference group for the odds ratio is achieving maximal viral suppression of HIV RNA $<75$ copies/ml at the end of the stabilization period on the current regimen.

${ }^{\mathrm{b}} \mathrm{New}$ class includes integrase inhibitors and entry inhibitors.

A total of 3419 subjects were included in the multivariable analysis; 28 were excluded due to missing CD4 cell count at regimen switch. MSM, men who have sex with men; NNRTI, nonnucleoside reverse transcriptase inhibitor; PI, protease inhibitor; NRTI, nucleoside reverse transcriptase inhibitor; cART, combination antiretroviral therapy.

was also protective for advanced virologic failure when compared with a PI-based regimen $[\mathrm{OR}=0.29(0.09-0.93)]$.

\section{Discussion}

We found that among a treatment-experienced HIV-infected patient population undergoing regimen switch, about $24 \%$ failed to achieve maximal viral suppression after 6 months on the new regimen. Furthermore, 12\% experienced advanced virologic failure. Adjusting for medication adherence, younger age, heterosexual patients compared with MSM, lower CD4 cell count, NRTI-only regimens compared with PI-based regimens, and previous virologic failure remained independent risk factors for advanced 
virologic failure. CART regimens based on new class or mixed class were protective for failing with LLV. In addition, we found that rates of treatment failure decrease as calendar year advanced, an observation reported by others as well. ${ }^{15,16}$

cART regimen level factors are important predictors for HIV virologic response. The likelihood of failing the current regimen increases as the patient's previous exposure to cART regimens increases, despite adjusting for previous treatment failure, current mediation adherence, and current ART class. This finding suggests other factors continue to remain important causes of virologic failure in patients failing previous regimens. As previously hypothesized by many researchers, treatment-experienced patients are more likely to harbor HIV virus undergoing accumulation of resistance and cross-resistance, which despite optimal clinician use of resistance tests increases the chance of failure for any given new regimen. However, during the time period examined several new classes of medications came into clinical use, which should result in improved outcomes in patients with resistant virus. Indeed, we found that regimens based on new classes of ART, such as integrase inhibitors and entry inhibitors, were associated with lower likelihood of failing with LLV. For patients with CD4 cell count of $200 / \mu \mathrm{l}$ and greater, new classes of antiretrovirals were also protective for advanced virologic failure. As expected, the NRTI-only regimens, to which only a small number of subjects $(n=54)$ switched, was a strong predictor for treatment failure.

Our finding that virologic failure at previous regimens was a strong predictor for future virologic failure was consistent with previous studies in the notion that the patient's history of viral suppression is a strong predictor for future virologic response. In the study by Reekie et al. virologic failure after a treatment change was associated with viral rebound in the year prior, as well as the time spent on maximal viral suppression. ${ }^{4}$ Several other studies also suggested that previous viral rebound, ${ }^{2}$ low-level viremia, ${ }^{5}$ as well as time since last virologic failure ${ }^{6,8}$ are all predictors for future viral rebound or virologic failure. Benzie and colleagues further reported that patients who ever experienced treatment failure would need to remain maximally viral suppressed for over 4 years to achieve a similar rate of viral rebound as those who never experienced treatment failure. ${ }^{6}$

Previous studies reported racial healthcare disparity in patients living with HIV infection. ${ }^{17,18}$ Mugavero and colleagues found that adherence to doctor's appointment largely explained the disparity in risk of virologic failure between African-Americans and whites. ${ }^{3}$ Similarly, in our study, after adjusting for medication adherence and other potential risk factors, racial/ethnic minorities did not have a statistically significantly elevated odds of treatment failure. Furthermore, it should be noted that given similar access to care, racial/ ethnic minority did not appear to have more adverse clinical events despite lower medication adherence, as shown in our previous study among KP patients initiating cART. ${ }^{19}$ However, as racial/ethnic minorities do have poorer adherence to regular follow-up visits and/or antiviral medications, ${ }^{3,19}$ adherence counseling and/or specialized pharmacist consultation should still be emphasized with these patients.

Several limitations should be considered when interpreting our results. Due to the observational nature of this study, there were no standardized follow-up visits, and a consider- able proportion of eligible patients (21\%) was excluded due to the lack of an HIV RNA measurement to determine virologic response on the new regimen. This could potentially lead to selection bias if patients lacking HIV RNA measurement were systemically different from other eligible patients. Furthermore, information on reasons for changing regimens for each individual was not available from electronic medical records and therefore was not totally accounted for in the analysis. In addition, we assessed only short-term virologic response on the new regimen. Since those who achieved maximal viral suppression at 6 months may experience viral rebound at a later point in time, our results do not apply to long-term maintenance of maximal viral control. Despite these limitations, our study had the strengths of a well-defined, racial/ ethnically diverse patient population, with the use of a comprehensive clinical record system that allows detailed assessment of each patient's' clinical history.

In conclusion, we found that a history of viral suppression, ART class, and number of cART regimens the patient is exposed to are among the strongest risk factors for future treatment failure. Use of a new class of antiretrovirals, medication adherence, and higher CD4 cell count, on the other hand, were predictors for achieving maximal viral suppression. These findings point to the importance of the choice of the new regimen, as well as maintaining CD4 cell count and maximal viral suppression. The risk factors identified in this study should be taken into consideration when considering a change of antiviral treatment and the subsequent patient care plan.

\section{Acknowledgments}

This study is supported by a research grant funded by Merck \& Co. MJS was supported by grant number K01AI071725 from the NIAID.

\section{Author Disclosure Statement}

The authors have received research funds from Merck \& Co and Pfizer, Inc. for other related research projects.

\section{References}

1. Guidelines for the Use of Antiretroviral Agents in HIV-1Infected Adults and Adolescents. 1-166. Available at http:// www.aidsinfo.nih.gov/ContentFiles/AdultandAdolescentGL .pdf. Accessed Aug 31, 2011.

2. Easterbrook PJ, Ives N, Waters A, et al.: The natural history and clinical significance of intermittent viraemia in patients with initial viral suppression to $<400$ copies/ml. AIDS 2002; 16(11):1521-1527.

3. Mugavero MJ, Lin HY, Allison JJ, et al.: Racial disparities in HIV virologic failure: Do missed visits matter? J Acquir Immune Defic Syndr 2009;50(1):100-108.

4. Reekie J, Mocroft A, Ledergerber B, et al.: History of viral suppression on combination antiretroviral therapy as a predictor of virological failure after a treatment change. HIV Med 2010;11(7):469-478.

5. Sungkanuparph S, Groger RK, Overton ET, Fraser VJ, and Powderly WG: Persistent low-level viraemia and virological failure in HIV-1-infected patients treated with highly active antiretroviral therapy. HIV Med 2006;7(7):437-441.

6. Benzie AA, Bansi LK, Sabin CA, et al.: Increased duration of viral suppression is associated with lower viral rebound 
rates in patients with previous treatment failures. AIDS 2007;21(11):1423-1430.

7. Paredes R, Mocroft A, Kirk O, et al.: Predictors of virological success and ensuing failure in HIV-positive patients starting highly active antiretroviral therapy in Europe: Results from the EuroSIDA study. Arch Intern Med 2000;160(8):1123-1132.

8. Phillips AN, Staszewski S, Lampe F, et al.: Human immunodeficiency virus rebound after suppression to $<400$ copies/mL during initial highly active antiretroviral therapy regimens, according to prior nucleoside experience and duration of suppression. J Infect Dis 2002;186(8):1086-1091.

9. Horberg M, Silverberg M, Hurley L, Delorenze G, and Quesenberry C: Influence of prior antiretroviral experience on adherence and responses to new highly active antiretroviral therapy regimens. AIDS Patient Care STDS 2008;22(4):301-312.

10. Paterson DL, Swindells S, Mohr J, et al.: Adherence to protease inhibitor therapy and outcomes in patients with HIV infection. Ann Intern Med 2000;133(1):21-30.

11. Oette M, Kroidl A, Gobels K, et al.: Predictors of short-term success of antiretroviral therapy in HIV infection. J Antimicrob Chemother 2006;58(1):147-153.

12. Murri R, Lepri AC, Cicconi P, et al.: Is moderate HIV viremia associated with a higher risk of clinical progression in HIVinfected people treated with highly active antiretroviral therapy: Evidence from the Italian cohort of antiretroviralnaive patients study. J Acquir Immune Defic Syndr 2006; 41(1):23-30.

13. Raffanti SP, Fusco JS, Sherrill BH, et al.: Effect of persistent moderate viremia on disease progression during HIV therapy. J Acquir Immune Defic Syndr 2004;37(1):1147-1154.

14. Ledergerber B, Egger M, Opravil M, et al.: Clinical progression and virological failure on highly active antiretroviral therapy in HIV-1 patients: A prospective cohort study. Swiss HIV Cohort Study. Lancet 1999;353(9156):863-868.

15. Moore RD, Keruly JC, Gebo KA, and Lucas GM: An improvement in virologic response to highly active antiretroviral therapy in clinical practice from 1996 through 2002. J Acquir Immune Defic Syndr 2005;39(2):195-198.

16. Deeks SG, Gange SJ, Kitahata MM, et al.: Trends in multidrug treatment failure and subsequent mortality among antiretroviral therapy-experienced patients with HIV infection in North America. Clin Infect Dis 2009;49(10):15821590.

17. Moore RD, Stanton D, Gopalan R, and Chaisson RE: Racial differences in the use of drug therapy for HIV disease in an urban community. N Engl J Med 1994;330(11):763-768.

18. Lillie-Blanton M, Stone VE, Snow Jones A, et al.: Association of race, substance abuse, and health insurance coverage with use of highly active antiretroviral therapy among HIVinfected women, 2005. Am J Public Health 2010;100(8):14931499.

19. Silverberg MJ, Leyden W, Quesenberry CP Jr, and Horberg MA: Race/ethnicity and risk of AIDS and death among HIV-infected patients with access to care. J Gen Intern Med 2009;24(9):1065-1072.

Address correspondence to:

Chun Chao

Department of Research and Evaluation

Kaiser Permanente Southern California

100 South Los Robles Avenue, 2nd Floor

Pasadena, California 91101

E-mail: chun.r.chao@kp.org 